\title{
A Facile Synthesis of Polysubstituted Pyrroles by One- Pot Three-Component Reaction
}

\author{
HOSSEIN ANARAKI-ARDAKANI ${ }^{1}$, MAZIAR NOEI ${ }^{1}$, MINA KARBALAEI- \\ HAROFTEH $^{2}$, SHAHAB ZOMORODBAKSH ${ }^{1}$ \\ ${ }^{1}$ Department of Chemistry, Mahshahr Branch, Islamic Azad University, Mahshahr, \\ Iran \\ ${ }^{2}$ Department of Chemistry, Islamic Azad University, Yazd Branch, P.O. Box 89195- \\ 155, Yazd, Iran \\ hosseinanaraki@yahoo.com
}

Received 28 October 2011; Acceptance 29 December 2011

\begin{abstract}
A new and efficient one-pot synthesis of polysubstituted pyrrole derivatives by three-component reaction between dialkyl acetylenedicarboxylates, triphenylphosphine, 2-aminopyridin derivatives in the presence of arylglyoxals is described. The reactions were performed in dichloromethane at room temperature and neutral conditions and afforded high yields of products.
\end{abstract}

Keywords: pyrrole, dialkyl acetylenedicarboxylates, 2-aminopyridin, triphenylphosphine, intramolecular Wittig reaction.

\section{Introduction}

Pyrroles ${ }^{1}$ are among the most important heterocyclic compounds as they are structural elements of various bioactive natural products ${ }^{2-4}$ and pharmaceutical agents. ${ }^{5-7}$ They are also valuable intermediates in organic synthesis. ${ }^{2,8}$ Various pyrrole derivatives are widely used as organic conducting materials. ${ }^{9}$ Consequently, a wide range of procedures have been devised for the synthesis of pyrroles. ${ }^{10-13}$ However, many of the methods are associated with various drawbacks such as harsh reaction conditions, tedious experimental procedures, unsatisfactory yields, and long reaction times. Moreover, the number of methods for the synthesis of polysubstituted pyrroles is relatively limited.

Addition reaction between phosphines and activated carbon-carbon triple bonds is well known to produce a reactive zwitterionic intermediate, which may be trapped by various electrophiles. ${ }^{14-18}$ Reaction of triphenylphosphine with dialkyl acetylenedicarboxylates (DAAD's) has been studied in the presence of a variety of organic acidic compounds, in order to trapp the zwitterionic intermediate. Trapping of $\mathrm{PPh}_{3}$-DAAD zwitterion by an organic acidic compound containing a carbonyl group have been used as a one-pot and efficient route for the synthesis of a variety of heterocylclic and carbocyclic compounds. ${ }^{19-23}$ 
Keeping in mind the biological importance of pyrrole ring and in continuation of our current studies on the development of new routes in heterocyclic synthesis, ${ }^{23-24}$ in this letter we report a simple and efficient synthesis of some functionalized pyrrole. Thus, threecomponent reaction between triphenylphosphine 1, 2-aminopyridin derivative 2, dialkyl acetylenedicarboxylates $\mathbf{3}$ in the presence of arylglyoxals $\mathbf{4}$ at ambient temperature in dichloromethane, leads to substituted pyrrole derivatives $\mathbf{5}$ in good yields (Figure 1).

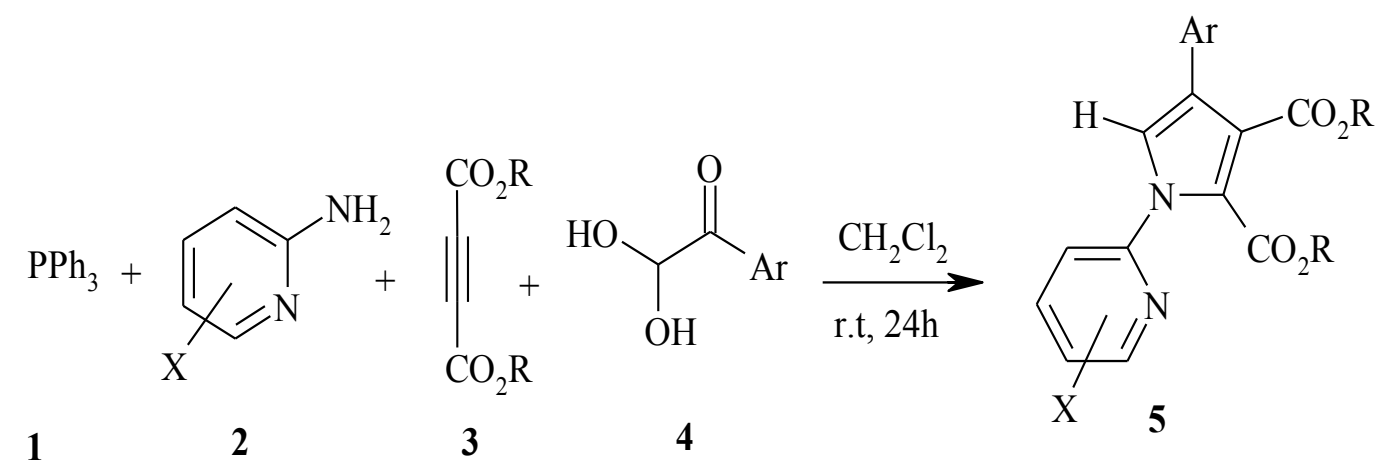

Figure 1. One-pot synthesis of some polyfunctionalized pyrroles.

\section{Experimental}

Melting points were determined with an electrothermal 9100 apparatus. Elemental analyses were performed using a Heraeus CHN-O-Rapid analyzer. Mass spectra were recorded on a FINNIGAN-MAT 8430 mass spectrometer operating at an ionization potential of $70 \mathrm{eV}$. IR spectra were recorded on a Shimadzu IR-470 spectrometer. ${ }^{1} \mathrm{H}$ and ${ }^{13} \mathrm{C}$ NMR spectra were recorded on Bruker DRX-500 Avance spectrometer at solution in $\mathrm{CDCl}_{3}$ using TMS as internal standard. The chemicals used in this work purchased from Fluka (Buchs, Switzerland) and were used without further purification.

\section{General Procedure}

To a magnetically stirred solution of triphenylphosphine $(1 \mathrm{mmol})$ and 2-aminopyridin derivatives $(1 \mathrm{mmol})$ in dichloromethane $(10 \mathrm{~mL})$ was added dropwise a mixture of dialkyl acetylenedicarboxylate $(1 \mathrm{mmol})$ in dichloromethane $(3 \mathrm{~mL})$ at room temperature over 5 min. The reaction mixture was then stirred for 10 minute. Arylglyoxal $(1 \mathrm{mmol})$ was added and the reaction mixture was stirred for more 24 hours. Solvent was evaporated and the residue was purified by column chromatography on silica-gel using ethyl acetate-hexane (1:4) mixture as eluent.

\section{Spectral data}

Dimethyl 1-(5-bromopyridin-2-yl)-4-(4-nitrophenyl)-1H-pyrrole-2,3-dicarboxylate (4a):

Yellow oil $(0.41 \mathrm{~g}, 90 \%)$. IR $(\mathrm{KBr})\left(v_{\max }, \mathrm{cm}^{-1}\right): 1715(\mathrm{C}=\mathrm{O}) .{ }^{1} \mathrm{H}$ NMR $(500.1 \mathrm{MHz}$, $\left.\mathrm{CDCl}_{3}\right) \delta=3.85\left(3 \mathrm{H}, \mathrm{s}, \mathrm{OCH}_{3}\right), 3.88\left(3 \mathrm{H}, \mathrm{s}, \mathrm{OCH}_{3}\right), 7.35(1 \mathrm{H}, \mathrm{s}, \mathrm{CH}), 7.33\left(1 \mathrm{H}, \mathrm{d},{ }^{3} \mathrm{~J}_{\mathrm{HH}}=\right.$ $8.5 \mathrm{~Hz}, 1 \mathrm{CH}$ of $\left.4-\mathrm{Br} \mathrm{C}_{5} \mathrm{H}_{3} \mathrm{~N}\right), 7.65\left(2 \mathrm{H}, \mathrm{d},{ }^{3} \mathrm{~J}_{\mathrm{HH}}=8.8 \mathrm{~Hz}, 2 \mathrm{CH}\right.$ of $\left.4-\mathrm{NO}_{2} \mathrm{C}_{6} \mathrm{H}_{4}\right), 7.99(1 \mathrm{H}$, $\mathrm{dd},{ }^{3} J_{\mathrm{HH}}=8.5 \mathrm{~Hz},{ }^{4} J_{\mathrm{HH}}=2.4 \mathrm{~Hz}, 1 \mathrm{CH}$ of $\left.4-\mathrm{Br} \mathrm{C}_{5} \mathrm{H}_{3} \mathrm{~N}\right), 8.26\left(2 \mathrm{H}, \mathrm{d},{ }^{3} J_{\mathrm{HH}}=8.8 \mathrm{~Hz}, 2 \mathrm{CH}\right.$ of $\left.4-\mathrm{NO}_{2} \mathrm{C}_{6} \mathrm{H}_{4}\right), 8.61\left(1 \mathrm{H}, \mathrm{d},{ }^{3} \mathrm{~J}_{\mathrm{HH}}=2.4 \mathrm{~Hz}, 1 \mathrm{CH}\right.$ of $\left.4-\mathrm{Br}_{5} \mathrm{H}_{3} \mathrm{~N}\right) \mathrm{ppm} .{ }^{13} \mathrm{C}$ NMR $(125.7$ $\left.\mathrm{MHz}, \mathrm{CDCl}_{3}\right): \delta=52.77\left(\mathrm{OCH}_{3}\right), 53.03\left(\mathrm{OCH}_{3}\right), 119.55,120.30,121.05,123.90,124.12$, $124.79,129.33,130.11,140.26,141.50,147.40149 .41,150.40$ (C arom), $161.53\left(\mathrm{CO}_{2} \mathrm{Me}\right)$, 
$165.25\left(\mathrm{CO}_{2} \mathrm{Me}\right)$. MS (m/z, \%): $459\left(\mathrm{M}^{+}\right.$, 5). Anal. Calcd. for $\mathrm{C}_{19} \mathrm{H}_{14} \mathrm{BrN}_{3} \mathrm{O}_{6}$ (459): $\mathrm{C}, 49.58$; H, 3.07; N, 9.13\%. Found: C, 49.32; H, 3.18; N, 9.29\%.

Diethyl 1-(5-bromopyridin-2-yl)-4-(4-nitrophenyl)-1H-pyrrole-2,3-dicarboxylate (4b):

Yellow oil $(0.41 \mathrm{~g}, 85 \%)$. IR $(\mathrm{KBr})\left(v_{\max }, \mathrm{cm}^{-1}\right): 1726(\mathrm{C}=\mathrm{O}) .{ }^{1} \mathrm{H}$ NMR $(500.1 \mathrm{MHz}$, $\left.\mathrm{CDCl}_{3}\right) \delta=\delta=1.32\left(6 \mathrm{H}, \mathrm{m}, 2 \mathrm{OCH}_{2} \mathrm{CH}_{3}\right), 4.22\left(2 \mathrm{H}, \mathrm{q},{ }^{3} J_{\mathrm{HH}}=7.1 \mathrm{~Hz}, \mathrm{OCH}_{2} \mathrm{CH}_{3}\right), 4.33$ $\left(4 \mathrm{H}, \mathrm{m}, 2 \mathrm{OCH}_{2} \mathrm{CH}_{3}\right), 7.33(1 \mathrm{H}, \mathrm{s}, \mathrm{CH}), 7.34\left(1 \mathrm{H}, \mathrm{d},{ }^{3} \mathrm{~J}_{\mathrm{HH}}=8.5 \mathrm{~Hz}, 1 \mathrm{CH}\right.$ of $\left.4-\mathrm{Br} \mathrm{C}_{5} \mathrm{H}_{3} \mathrm{~N}\right)$, $7.65\left(2 \mathrm{H}, \mathrm{d},{ }^{3} J_{\mathrm{HH}}=8.8 \mathrm{~Hz}, 2 \mathrm{CH}\right.$ of $\left.4-\mathrm{NO}_{2} \mathrm{C}_{6} \mathrm{H}_{4}\right), 7.99\left(1 \mathrm{H}, \mathrm{dd},{ }^{3} J_{\mathrm{HH}}=8.5 \mathrm{~Hz},{ }^{4} J_{\mathrm{HH}}=2.4 \mathrm{~Hz}\right.$, $1 \mathrm{CH}$ of $\left.4-\mathrm{Br} \mathrm{C}_{5} \mathrm{H}_{3} \mathrm{~N}\right), 8.26\left(2 \mathrm{H}, \mathrm{d},{ }^{3} J_{\mathrm{HH}}=8.8 \mathrm{~Hz}, 2 \mathrm{CH}\right.$ of $\left.4-\mathrm{NO}_{2} \mathrm{C}_{6} \mathrm{H}_{4}\right), 8.60\left(1 \mathrm{H}, \mathrm{d},{ }^{3} J_{\mathrm{HH}}\right.$ $=2.4 \mathrm{~Hz}, 1 \mathrm{CH}$ of $\left.4-\mathrm{Br} \mathrm{C}_{5} \mathrm{H}_{3} \mathrm{~N}\right) \mathrm{ppm} .{ }^{13} \mathrm{C} \mathrm{NMR}\left(125.7 \mathrm{MHz}, \mathrm{CDCl}_{3}\right): \delta=14.37,14.44$ $\left(2 \mathrm{OCH}_{2} \mathrm{CH}_{3}\right), 61.85,62.13\left(2 \mathrm{OCH}_{2} \mathrm{CH}_{3}\right), 119.79,120.25,121.43,123.88,124.08,124.62$, $129.31,131.30,140.40,141.41,147.34,149.55,150.30(\mathrm{C}$ arom $), 161.01\left(\mathrm{CO}_{2} \mathrm{Me}\right), 164.91$ $\left(\mathrm{CO}_{2} \mathrm{Me}\right)$. MS (m/z, \%): 487( $\left.\mathrm{M}^{+}, 7\right)$. Anal. Calcd. for $\mathrm{C}_{21} \mathrm{H}_{18} \mathrm{BrN}_{3} \mathrm{O}_{6}(487)$ : C, 51.65; H, 3.72 ; N, 8.61\%. Found: C, 51.52; H, 3.62; N, 8.86\%.

Dimethyl 4-(4-bromophenyl)-1-(5-chloropyridin-2-yl)-1H-pyrrole-2,3-dicarboxylate (4c):

Yellow oil $(0.35 \mathrm{~g}, 80 \%)$. IR $(\mathrm{KBr})\left(v_{\max }, \mathrm{cm}^{-1}\right): 1721(\mathrm{C}=\mathrm{O}) .{ }^{1} \mathrm{H}$ NMR $(500.1 \mathrm{MHz}$, $\left.\mathrm{CDCl}_{3}\right) \delta=3.83\left(3 \mathrm{H}, \mathrm{s}, \mathrm{OCH}_{3}\right), 3.84\left(3 \mathrm{H}, \mathrm{s}, \mathrm{OCH}_{3}\right), 7.32(1 \mathrm{H}, \mathrm{s}, \mathrm{CH}), 7.35\left(1 \mathrm{H}, \mathrm{d},{ }^{3} \mathrm{~J}_{\mathrm{HH}}=\right.$ $8.5 \mathrm{~Hz}, 1 \mathrm{CH}$ of $\left.4-\mathrm{Cl} \mathrm{C}_{5} \mathrm{H}_{3} \mathrm{~N}\right), 7.36\left(2 \mathrm{H}, \mathrm{d},{ }^{3} J_{\mathrm{HH}}=8.3 \mathrm{~Hz}, 2 \mathrm{CH}\right.$ of $\left.4-\mathrm{BrC}_{6} \mathrm{H}_{4}\right), 7.51(2 \mathrm{H}, \mathrm{d}$, ${ }^{3} J_{\mathrm{HH}}=8.3 \mathrm{~Hz}, 2 \mathrm{CH}$ of $\left.4-\mathrm{BrC}_{6} \mathrm{H}_{4}\right), 7.85\left(1 \mathrm{H}, \mathrm{dd},{ }^{3} J_{\mathrm{HH}}=8.5 \mathrm{~Hz},{ }^{4} J_{\mathrm{HH}}=2.4 \mathrm{~Hz}, 1 \mathrm{CH}\right.$ of $4-\mathrm{Cl}$ $\left.\mathrm{C}_{5} \mathrm{H}_{3} \mathrm{~N}\right), 8.48\left(1 \mathrm{H}, \mathrm{d},{ }^{3} \mathrm{~J}_{\mathrm{HH}}=2.4 \mathrm{~Hz}, 1 \mathrm{CH}\right.$ of $\left.4-\mathrm{Cl} \mathrm{C}_{5} \mathrm{H}_{3} \mathrm{~N}\right) \mathrm{ppm} .{ }^{13} \mathrm{C}$ NMR $(125.7 \mathrm{MHz}$, $\left.\mathrm{CDCl}_{3}\right): \delta=52.63\left(\mathrm{OCH}_{3}\right), 52.87\left(\mathrm{OCH}_{3}\right), 119.32,121.94,121.05,123.52,124.12,125.05$, $125.67,128.75,130.27,131.94,137.97,138.51,139.10,147.48,148.00,149.23$ (C arom), $161.62\left(\mathrm{CO}_{2} \mathrm{Me}\right)$, 65.71 $\left(\mathrm{CO}_{2} \mathrm{Me}\right)$. MS (m/z, \%): 447( $\mathrm{M}^{+}$, 9). Anal. Calcd. for $\mathrm{C}_{19} \mathrm{H}_{14} \mathrm{BrClN}_{2} \mathrm{O}_{4}$ (447): C, 50.75; H, 3.14; N, 6.23\%. Found: C, 50.62; H, 3.31; N, 6.12\%.

Dimethyl 1-(5-chloropyridin-2-yl)-4-(4-nitrophenyl)-1H-pyrrole-2,3-dicarboxylate(4d):

Yellow oil $(0.35 \mathrm{~g}, 85 \%)$. IR $(\mathrm{KBr})\left(v_{\max }, \mathrm{cm}^{-1}\right): 1709(\mathrm{C}=\mathrm{O}) .{ }^{1} \mathrm{H}$ NMR $(500.1 \mathrm{MHz}$, $\left.\mathrm{CDCl}_{3}\right) \delta=3.87\left(3 \mathrm{H}, \mathrm{s}, \mathrm{OCH}_{3}\right), 3.85\left(3 \mathrm{H}, \mathrm{s}, \mathrm{OCH}_{3}\right), 7.29(1 \mathrm{H}, \mathrm{s}, \mathrm{CH}), 7.38\left(1 \mathrm{H}, \mathrm{d},{ }^{3} \mathrm{~J}_{\mathrm{HH}}=\right.$ $8.5 \mathrm{~Hz}, 1 \mathrm{CH}$ of $\left.4-\mathrm{Cl} \mathrm{C}_{5} \mathrm{H}_{3} \mathrm{~N}\right), 7.64\left(2 \mathrm{H}, \mathrm{d},{ }^{3} \mathrm{~J}_{\mathrm{HH}}=8.7 \mathrm{~Hz}, 2 \mathrm{CH}\right.$ of $\left.4-\mathrm{NO}_{2} \mathrm{C}_{6} \mathrm{H}_{4}\right), 7.85(1 \mathrm{H}$, $\mathrm{dd},{ }^{3} J_{\mathrm{HH}}=8.5 \mathrm{~Hz},{ }^{4} J_{\mathrm{HH}}=2.4 \mathrm{~Hz}, 1 \mathrm{CH}$ of $\left.4-\mathrm{Cl} \mathrm{C}_{5} \mathrm{H}_{3} \mathrm{~N}\right), 8.26\left(2 \mathrm{H}, \mathrm{d},{ }^{3} J_{\mathrm{HH}}=8.7 \mathrm{~Hz}, 2 \mathrm{CH}\right.$ of $\left.4-\mathrm{NO}_{2} \mathrm{C}_{6} \mathrm{H}_{4}\right), 8.50\left(1 \mathrm{H}, \mathrm{d},{ }^{3} \mathrm{~J}_{\mathrm{HH}}=2.4 \mathrm{~Hz}, 1 \mathrm{CH}\right.$ of $\left.4-\mathrm{Cl} \mathrm{C}_{5} \mathrm{H}_{3} \mathrm{~N}\right) \mathrm{ppm} .{ }^{13} \mathrm{C}$ NMR $(125.7$ $\left.\mathrm{MHz}, \mathrm{CDCl}_{3}\right): \delta=52.80\left(\mathrm{OCH}_{3}\right), 53.05\left(\mathrm{OCH}_{3}\right), 119.18,120.320,121.14,124.02,124.14$, 124.73, 129.31, 132.05, 138.67, 140.26, 147.38, 148.14, 148.97 (C arom), $161.53\left(\mathrm{CO}_{2} \mathrm{Me}\right)$, $165.25\left(\mathrm{CO}_{2} \mathrm{Me}\right)$. MS (m/z, \%): 415(M $\left.\mathrm{M}^{+}, 6\right)$. Anal. Calcd. for $\mathrm{C}_{19} \mathrm{H}_{14} \mathrm{ClN}_{3} \mathrm{O}_{6}(415): \mathrm{C}, 54.89$; $\mathrm{H}, 3.39$; N, 10.11\%. Found: C, 54.72; H, 3.16; N, 10.21\%.

Dimethyl 4-(4-bromophenyl)-1-(4-methylpyridin-2-yl)-1H-pyrrole-2,3-dicarboxylate(4e):

Yellow oil $(0.34 \mathrm{~g}, 80 \%)$. IR $(\mathrm{KBr})\left(v_{\max }, \mathrm{cm}^{-1}\right): 1728(\mathrm{C}=\mathrm{O}) .{ }^{1} \mathrm{H}$ NMR $(500.1 \mathrm{MHz}$, $\left.\mathrm{CDCl}_{3}\right) \delta=2.41\left(3 \mathrm{H}, \mathrm{s}, \mathrm{CH}_{3}\right), 3.78\left(3 \mathrm{H}, \mathrm{s}, \mathrm{OCH}_{3}\right), 3.82\left(3 \mathrm{H}, \mathrm{s}, \mathrm{OCH}_{3}\right), 6.69\left(1 \mathrm{H}, \mathrm{d},{ }^{3} J_{\mathrm{HH}}\right.$ $\left.=5.2 \mathrm{~Hz}, 5-\mathrm{CH}_{3} \mathrm{C}_{5} \mathrm{H}_{3} \mathrm{~N}\right), 7.21(1 \mathrm{H}, \mathrm{s}, \mathrm{CH}), 7.36\left(2 \mathrm{H}, \mathrm{d},{ }^{3} \mathrm{~J}_{\mathrm{HH}}=8.3 \mathrm{~Hz}, 2 \mathrm{CH}\right.$ of $\left.4-\mathrm{BrC}_{6} \mathrm{H}_{4}\right)$, $7.51\left(2 \mathrm{H}, \mathrm{d},{ }^{3} J_{\mathrm{HH}}=8.3 \mathrm{~Hz}, 2 \mathrm{CH}\right.$ of $\left.4-\mathrm{BrC}_{6} \mathrm{H}_{4}\right), 7.96\left(1 \mathrm{H}, \mathrm{d},{ }^{3} \mathrm{~J}_{\mathrm{HH}}=5.2 \mathrm{~Hz}, 5-\mathrm{CH}_{3} \mathrm{C}_{5} \mathrm{H}_{3} \mathrm{~N}\right)$, $8.01\left(1 \mathrm{H}, \mathrm{s}, 5-\mathrm{CH}_{3} \mathrm{C}_{5} \mathrm{H}_{3} \mathrm{~N}\right)$, ppm. ${ }^{13} \mathrm{C}$ NMR $\left(125.7 \mathrm{MHz}, \mathrm{CDCl}_{3}\right): \delta=14.54\left(\mathrm{CH}_{3}\right), 52.45$ $\left(\mathrm{OCH}_{3}\right), 52.69\left(\mathrm{OCH}_{3}\right), 114.3,121.6,122.14,124.11,124.21,124.61,129.30,132.14$, 138.61, 140.22, 147.06, 149.61, 149.19 (C arom), $162.11\left(\mathrm{CO}_{2} \mathrm{Me}\right), 166.05\left(\mathrm{CO}_{2} \mathrm{Me}\right) . \mathrm{MS}$ (m/z, \%): $428\left(\mathrm{M}^{+}, 8\right)$. Anal. Calcd. for $\mathrm{C}_{20} \mathrm{H}_{17} \mathrm{BrN}_{2} \mathrm{O}_{4}$ (428): $\mathrm{C}, 55.96 ; \mathrm{H}, 3.99 ; \mathrm{N}, 6.53 \%$. Found: C, 55.82; H, 3.78; N, 6.81\%. 


\section{Results and Discussion}

We first prepared Ylide 6 by the reaction between triphenylphosphine, dimethyl acetylenedicarboxylate (DMAD) and 5-bromo-2-aminopyridin by the previously reported procedure. $^{25}$ (Figure 1). When phosphorane 6 was stirred with an equimolar amount of phenylglyoxal in dichloromethane, a smooth reaction took place. After completion of the reaction (monitored by TLC) dimethyl 1-(5-bromopyridin-2-yl)-4-(4-nitrophenyl)-1Hpyrrole-2,3-dicarboxylate 5a was obtained in $90 \%$ yield. As shown in Scheme 2, it is reasonable to assume that the Wittig reaction between phosphorane 4 and phenylglyoxal (5) affords intermediate $\mathbf{7}$ which then cyclizes to dihydropyrrole intermediate $\mathbf{8}$ that subsequently loses water to produce pyrrole derivative 5a.<smiles>CC(=O)C#CC(=O)OC(C)=O</smiles>

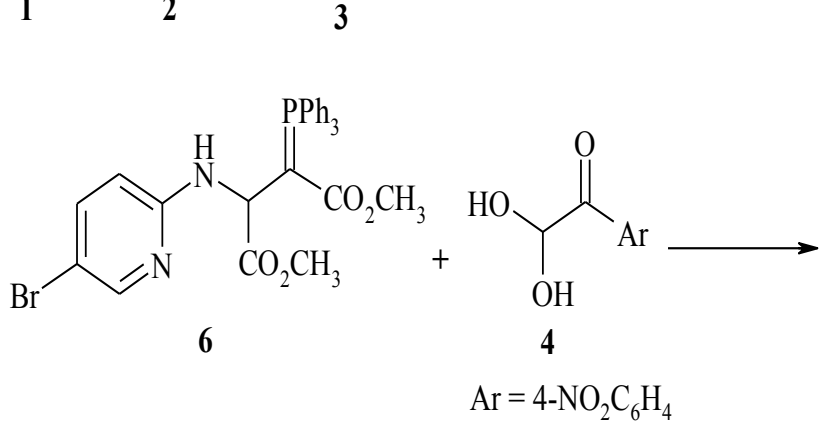<smiles>COC(=O)C(C(C)=O)N(c1ccc(Br)cn1)C(O)C(=O)[Mg]</smiles>

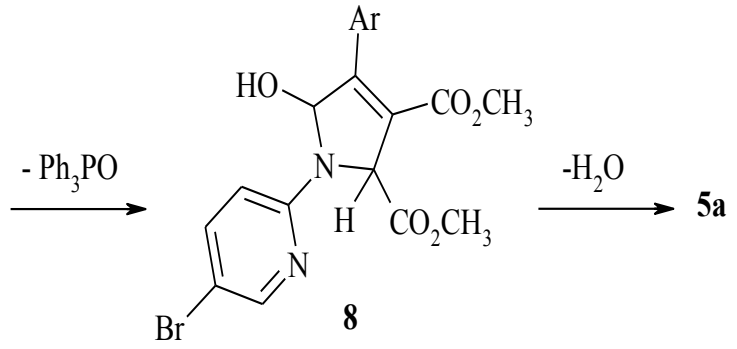

Figure 2 Suggested mechanism for formation of compound $\mathbf{5 a}$.

To explore the scope and limitations of this reaction further, we extended our studies to the reaction of various dialkyl acetylenedicarboxylates and 2-aminopyridin derivative with arylglyoxals (Table 1). As indicated in Table 1, the reactions proceeded very efficiently in relatively good yields. 
Table 1. Three-component reaction between 2-aminopyridin, DAAD's and arylglyoxals promoted by $\mathrm{PPh}_{3}$.

\begin{tabular}{c|cccc}
$\mathbf{5}$ & $\mathbf{R}$ & $\mathbf{A r}$ & $\mathbf{X}$ & Yield a \% \\
\hline $\mathbf{a}$ & $\mathrm{Me}$ & $4-\mathrm{NO}_{2} \mathrm{C}_{6} \mathrm{H}_{4}$ & $5-\mathrm{Br}$ & 90 \\
$\mathbf{b}$ & $\mathrm{Et}$ & $4-\mathrm{NO}_{2} \mathrm{C}_{6} \mathrm{H}_{4}$ & $5-\mathrm{Br}$ & 85 \\
c & $\mathrm{Me}$ & $4-\mathrm{BrC}_{6} \mathrm{H}_{4}$ & $5-\mathrm{Cl}$ & 80 \\
d & $\mathrm{Me}$ & $4-\mathrm{NO}_{2} \mathrm{C}_{6} \mathrm{H}_{4}$ & $5-\mathrm{Cl}$ & 85 \\
e & $\mathrm{Me}$ & $4-\mathrm{BrC}_{6} \mathrm{H}_{4}$ & $4-\mathrm{CH}_{3}$ & 80 \\
\hline
\end{tabular}

assolated Yield

The structures of compounds 5a-e were deduced from their elemental analyses and their IR, ${ }^{1} \mathrm{H}$ NMR, ${ }^{13} \mathrm{C}$ NMR spectra. The ${ }^{1} \mathrm{H}$ NMR spectrum 5a exhibited three sharp signals at $\delta$ $=3.85,3.88$ and $7.33 \mathrm{ppm}$ for two methoxy groups protons and the proton of pyrrole ring, respectively. The aryl moiety exhibited characteristic signals in the aromatic region of the spectrum. The ${ }^{13} \mathrm{C}$ NMR spectrum of compound 6 a showed 17 distinct resonances in agreement with the proposed structure.

\section{Conclusion}

In conclusion here we reported a three-component reaction between dialkyl acetylenedicarboxylates, 2-aminopyridin derivatives and arylglioxals promoted by triphenylphosphine, to produce functionalized pyrrole derivatives in high yields. The present method carries the advantage that, not only is the reaction performed under neutral conditions, but also the substances can be mixed without any activation or modification. The simplicity of the present procedure makes it an interesting alternative to complex multi-step approaches.

\section{Acknowledgements}

We gratefully acknowledge the financial support from the Research Council of Islamic Azad University, Mahshahr Branch.

\section{References}

1. Part 283 in the series 'Studies on Novel SyntheticMethodologies'.

2. Finar I L, Organic Chemistry: Stereochemistry and Chemistry of Natural Products, 5th ed., Vol. 2; Longman: London, 1975, 885-913.

3. 'Hagan D O, Nat Prod Rep., 2000, 17, 435.

4. Walsh C T, Garneau-Tsodikova S, Howard-Jones A R, Nat Prod Rep., 2006, 23, 517. 
5. Huffman J W, Curr Med Chem., 1999, 6, 705.

6. Kidwai M, Venkataramanan R, Mohan R, Sapra P, Curr Med Chem., 2002, 9, 1209.

7. LaRegina G, Silvestri R, Artico, Lavechia M, Novellino, A. Befani E, Turini O, Agostinelli E P, J Med Chem., 2007, 50, 922.

8. Abid M, Landge S M, Torok B, Org Prep Proced Int., 2006, 38, 495.

9. Facchetti A, Abboto A, Beverina L, vander Boom M E, Dutta P, Evmenenko G, Pagani G A, Marks T , J Chem Mater., 2003, 15, 1064.

10. Larionov O V, Meijere A, Angew Chem Int Ed., 2005, 44, 5664.

11. Minetto G, Raveglia L F, Sega A, Taddei M, Eur J Org Chem., 2005, 24, 5277.

12. Shiner C M, Laner T D, Tetrahedron, 2005, 61, 11628.

13. Abid M, Teixeira L, Torok B, Org Lett., 2008, 10, 933.

14. Anaraki-Ardakani H, Mosslemin M H, Mohebat R, Barazandeh-Doust M , J Chem Res., 2011, 1, 32

15. Trost B M, Dake G R, J Am Chem Soc., 1997, 119, 7595.

16. Anary-Abbasinejad M, Anaraki-Ardakani H, Hosseini-Mehdiabad H, Phosphorus, Sulfur and Silicon and the Rela. Elem., 2008, 183, 1440

17. Yavari I, Hekmat-Shoar R, Zonouzi A, Tetrahedron Lett., 1998, 39, 2391.

18. Evans L A, Griffiths K E, Guthmann H , Murphy P J, Tetrahedron Lett., 2002, 43, 299.

19. Khabazzadeh H, Saidi K, Sheibani H, Islami M R, Phosphorus, Sulfur and Silicon Relat. Elem, 2007, 182, 2163.

20. Anary-Abbasinejad M, Mosslemin M H, Hassanabadi A, Tabatabaee M, Synth Commun., 2008, 38, 3700.

21. Mosslemin M H, Yavari I, Anary-abbasinejad M, Nateghi M R, Synthesis, 2004, 7, 1029.

22. Nair V, Nair J S, Vinod A U, Rath N P, J Chem Soc., Perkin Trans 1, 1997, 329.

23. Anaraki-Ardakani H, Mosslemin M H, Anary-Abbasinejad M, Shams N, Mirhosseini S H, Arkivoc, 2010, (xi), 343.

24. Anary-Abbasinejad M, Anaraki-Ardakani H, Dehghan A, Hassanabadi A, Seyedmir M R, J Chem Res., 2007, 10, 574.

25. Yavari I, Adib M, Hojabri L, Tetrahedron, 2002, 58, 7213. 


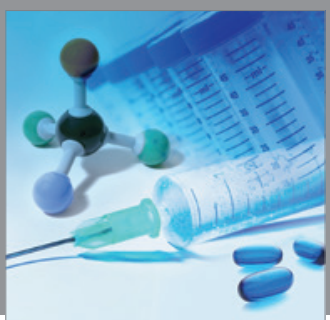

International Journal of

Medicinal Chemistry

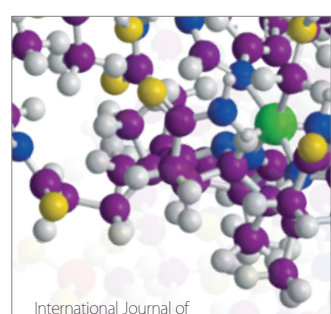

Carbohydrate Chemistry

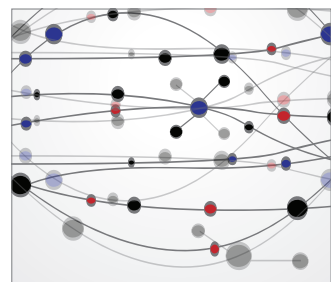

The Scientific World Journal
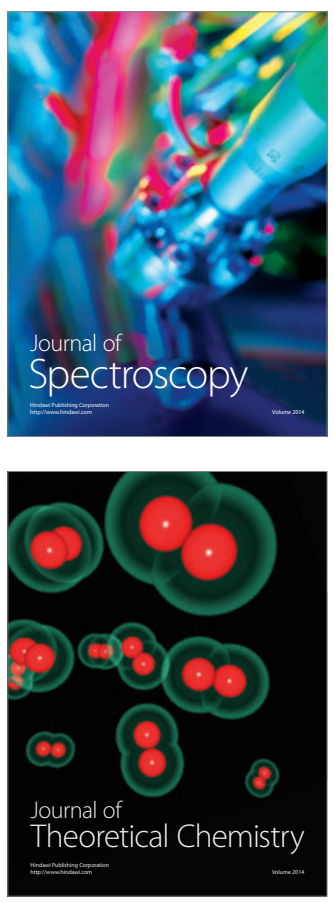
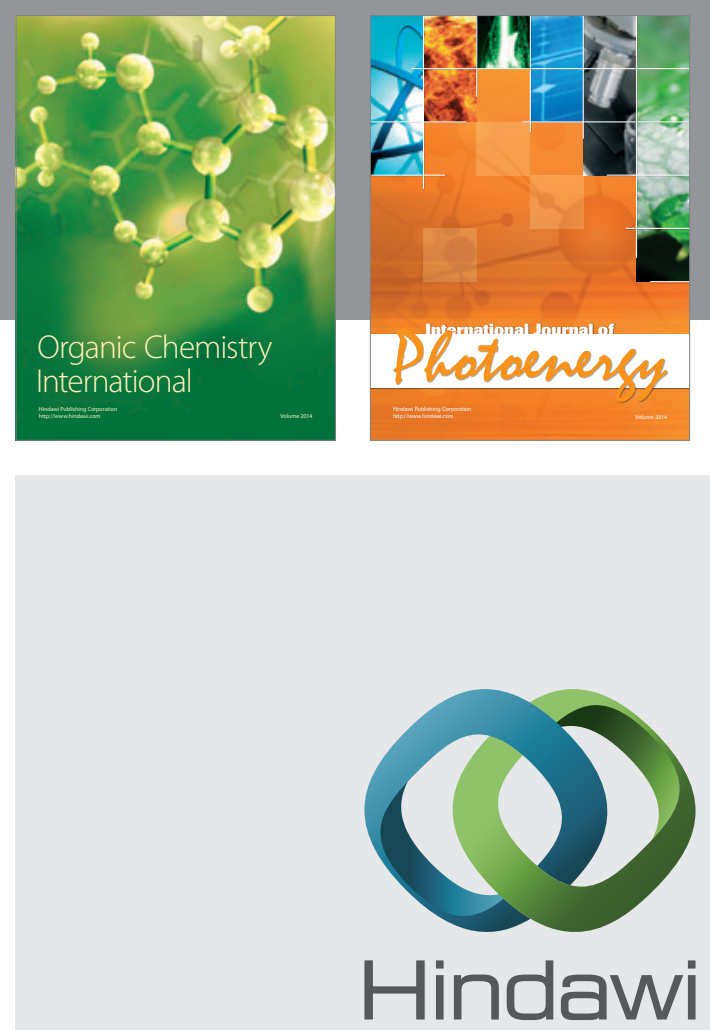

Submit your manuscripts at

http://www.hindawi.com
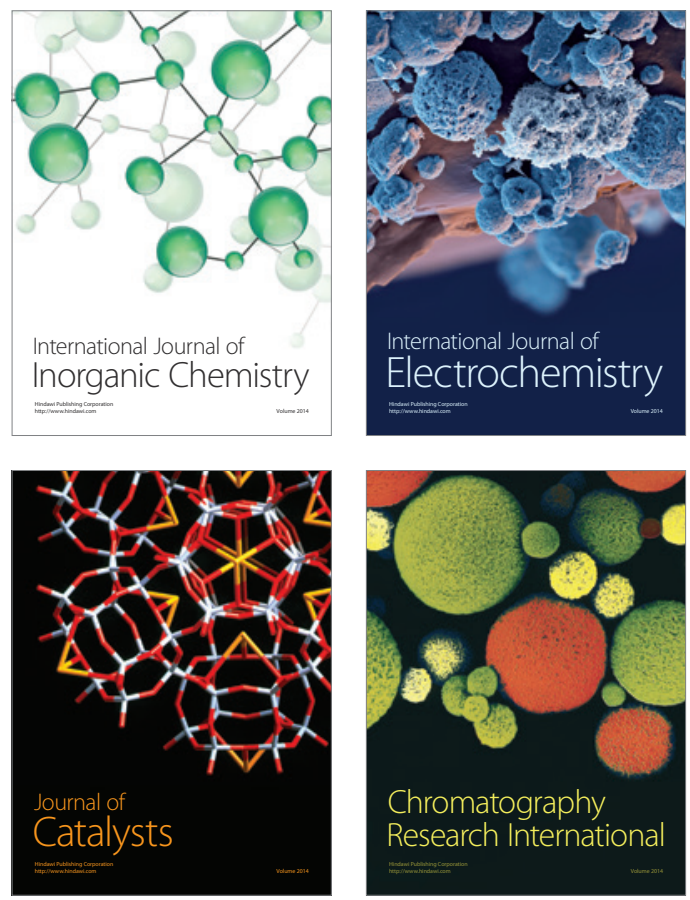
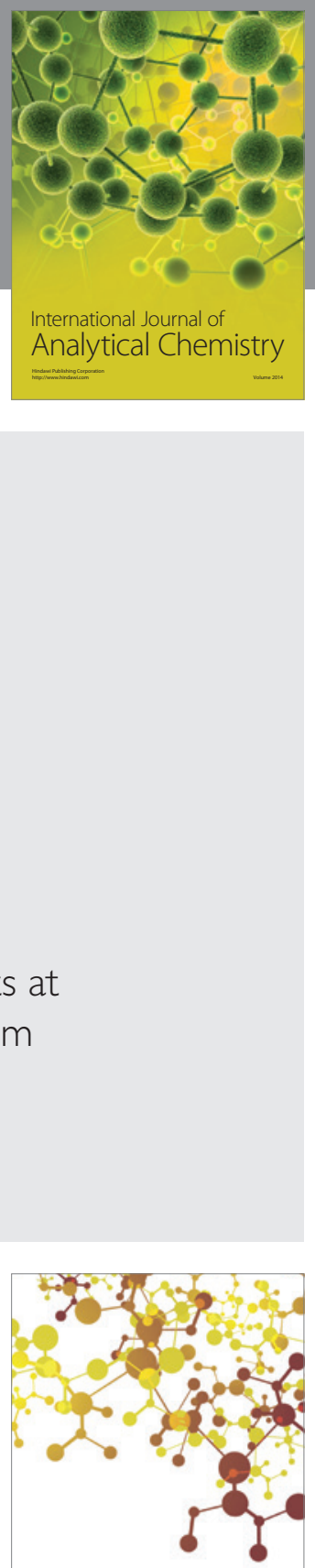

Journal of

Applied Chemistry
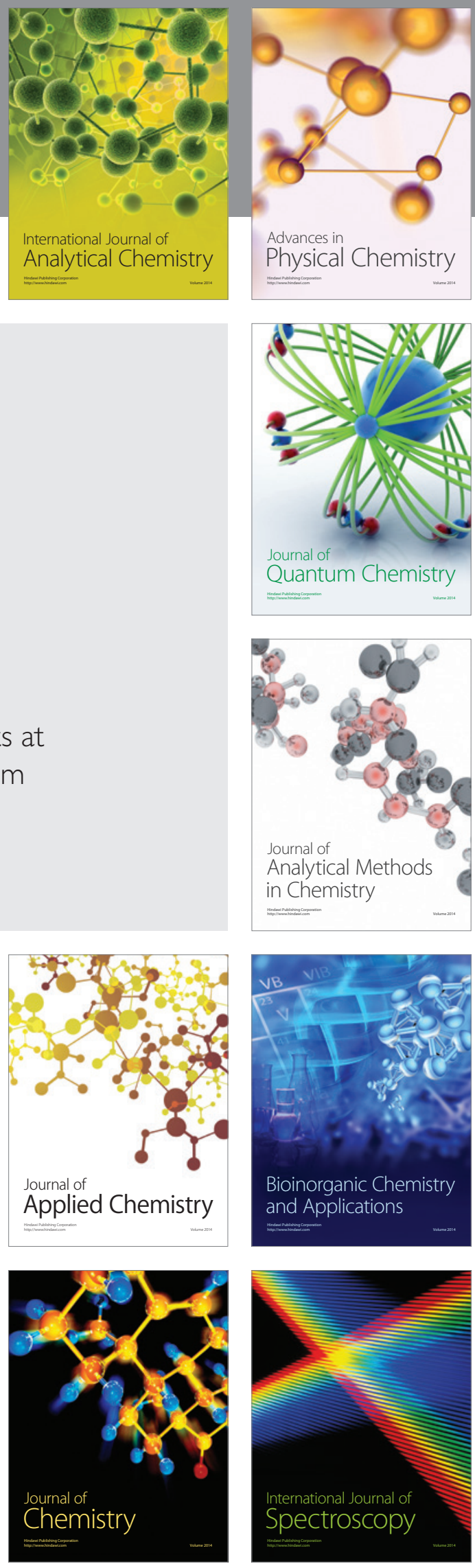